\title{
Insights into glycogen metabolism in Lactobacillus acidophilus: impact on carbohydrate metabolism, stress tolerance and gut retention
}

\author{
Yong Jun Goh ${ }^{*}$ and Todd R Klaenhammer
}

\begin{abstract}
In prokaryotic species equipped with glycogen metabolism machinery, the co-regulation of glycogen biosynthesis and degradation has been associated with the synthesis of energy storage compounds and various crucial physiological functions, including global cellular processes such as carbon and nitrogen metabolism, energy sensing and production, stress response and cell-cell communication. In addition, the glycogen metabolic pathway was proposed to serve as a carbon capacitor that regulates downstream carbon fluxes, and in some microorganisms the ability to synthesize intracellular glycogen has been implicated in host persistence. Among lactobacilli, complete glycogen metabolic pathway genes are present only in select species predominantly associated with mammalian hosts or natural environments. This observation highlights the potential involvement of glycogen biosynthesis in probiotic activities and persistence of intestinal lactobacilli in the human gastrointestinal tract. In this review, we summarize recent findings on (i) the presence and potential ecological distribution of glycogen metabolic pathways among lactobacilli, (ii) influence of carbon substrates and growth phases on glycogen metabolic gene expression and glycogen accumulation in L. acidophilus, and (iii) the involvement of glycogen metabolism on growth, sugar utilization and bile tolerance. Our present in vivo studies established the significance of glycogen biosynthesis on the competitive retention of L. acidophilus in the mouse intestinal tract, demonstrating for the first time that the ability to synthesize intracellular glycogen contributes to gut fitness and retention among probiotic microorganisms.
\end{abstract}

Keywords: Lactobacilli, Glycogen, Probiotic, Raffinose, Gastrointestinal retention

\section{Introduction}

Glycogen, a soluble multi-branched glucose homopolysaccharide, is a common form of energy storage synthesized by animals and eukaryotic microorganisms. Among prokaryotes, intracellular glycogen has been identified in more than 50 bacterial species including Gram-positive and Gram-negative bacteria as well as archaebacteria (reviewed in [1]). Based on a recent analysis of 1,202 bacterial genomes, intact glycogen metabolic pathways are generally present in species adaptable to more diverse habitats and flexible lifestyles [2]. Structurally analogous to amylopectin, an energy storage component of starch in plants, glycogen is composed of chains of $\alpha$-1,4-linked glucose units interconnected by comparatively more extensive $\alpha-1,6$-linked branches. Most bacterial glycogen has an

\footnotetext{
*Correspondence: yjgoh@ncsu.edu

Department of Food, Bioprocessing and Nutrition Sciences, North Carolina State University, Raleigh, North Carolina 27695, USA
}

average chain length of $\sim 7$ to 13 glucose units and an estimated molecular size of ca. $10^{7}$ to $10^{8} \mathrm{Da}[2,3]$. Glycogen is considered a flexible and efficient form of energy storage due to its large molecular mass and highly branched structure, and its accumulation has little effect on the internal osmotic pressure of the cells.

Glycogen synthesis in bacteria is generally regarded as a mechanism to prolong survival by supplying energy production during growth-limiting conditions. This so called "energy of maintenance" allows cells to sense and respond to nonsupportive environments, such as starvation and stress [4]. Glycogen may be synthesized during exponential growth or in stationary phase, and its accumulation generally occurs in the presence of excess carbon sources [4]. The classical biosynthetic pathway involves phosphoglucomutase (Pgm), glucose-1-phosphate adenylyltransferase (GlgC or GlgCD), ADP-glucose-specific glycogen synthase (GlgA) and branching enzyme (GlgB) 
(Figure 1). Phosphoglucomutase converts glucose-6phosphate into glucose-1-phosphate, which serves as a substrate for ADP-glucose synthesis catalyzed by GlgC or GlgCD. Then, GlgA catalyzes the transfer of glucosyl units from ADP-glucose to the elongating chain of linear $\alpha-1,4$-glucan. GlgB subsequently cleaves off portions of the glucan and links it to internal glucose molecules in existing chains via $\alpha-1,6$ glycosidic bonds to form the glycogen structure. The catabolism of glycogen is mediated by glycogen phosphorylase (GlgP) [5] and debranching enzyme (GlgX or Amy) [6], which catalyze the sequential phosphorolysis of $\alpha-1,4$-glucosyl linkages in the glucan chain from the non-reducing ends and debranching of the limit dextrins generated by GlgP, respectively.

Although the precise function of bacterial glycogen is not well-defined, an increasing number of studies have revealed the involvement of glycogen metabolism in major physiological roles, beyond the synthesis of energy reserve compounds (Table 1). Eydallin et al. showed that in Escherichia coli, glycogen metabolism is interconnected with global cellular processes including energy production, nutrient transport and metabolism, cell envelope integrity, protein turnover, stress responses and intercellular communication, and is tightly regulated by nutritional and energy status [7,8]. Glycogen synthesis also plays a role in the sporulation of Bacillus subtilis [9] and the biosynthesis of trehalose in Corynebacterium glutamicum which contributes to osmoprotection and cell wall synthesis [10,11]. In Mycobacterium smegmatis and several other microorganisms, the parallel synthesis and degradation of glycogen during early growth phase suggests that the glycogen metabolic pathway functions as a carbon capacitor that regulates downstream carbon and energy fluxes $[6,11,12]$. The ability to synthesize glycogen has also been associated with the colonization persistence of Streptococcus mutans [13], Mycobacterium tuberculosis [14] and E. coli [15], indicating glycogen synthesis as an important niche factor in the host environments.

Lactobacillus acidophilus was the first probiotic microorganism demonstrated to possess a functional glycogen biosynthetic pathway [23]. This ubiquitous probiotic microbe is widely used in the manufacture of yogurt, fermented dairy products and probiotic supplements [24]. Probiotic attributes of L. acidophilus include the alleviation of lactose intolerance and cold and influenza-like symptoms [25,26], the modulation of immune cell functions

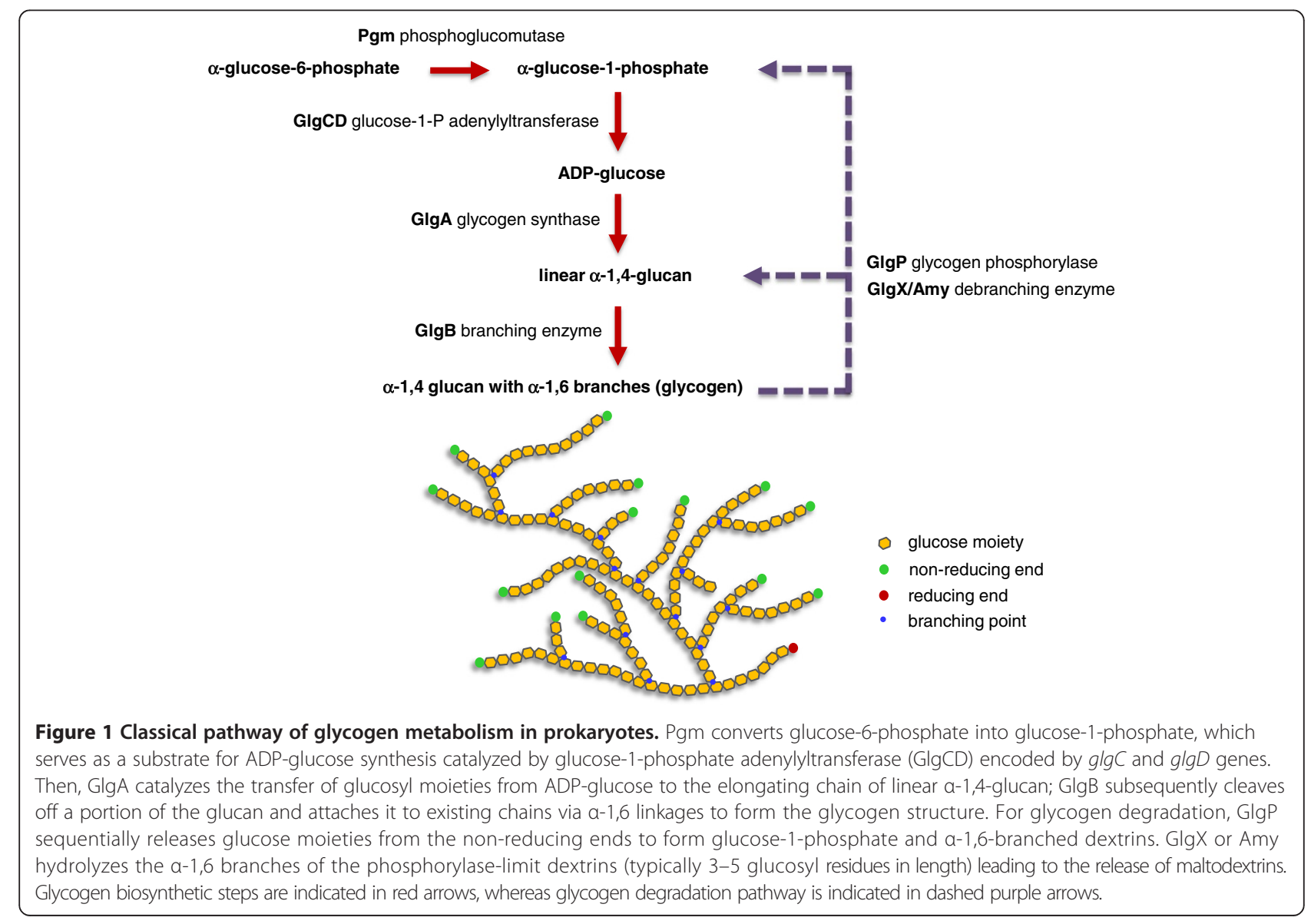


Table 1 Functional roles of glycogen metabolic pathway in bacteria

\begin{tabular}{lll}
\hline Species & Roles of glycogen storage/metabolism & References \\
\hline Bacillus subtilis & Sporulation & {$[9]$} \\
Corynebacterium glutamicum & Synthesis of trehalose, which is involved in cell wall synthesis and osmoprotection & {$[10,11,16]$} \\
Escherichia coli & Intestinal colonization in mice & {$[15]$} \\
Mycobacterium smegmatis & Carbon capacitor for glycolysis & {$[12]$} \\
Mycobacterium tuberculosis & Capsular glucan synthesis and persistence in mice & {$[14]$} \\
Propionibacterium freudenreichii & Long term survival and activity under low temperatures & {$[17]$} \\
Salmonella enteritidis & Biofilm formation and virulence & {$[18]$} \\
Streptococcus mutans & Persistence in oral cavity and formation of dental caries & {$[13,19,20]$} \\
Synechococcus elongatus & Tolerance to salt and oxidative stress & {$[21]$} \\
Vibrio cholerae & Environmental persistence and host transmission & {$[22]$} \\
\hline
\end{tabular}

[27] and the alleviation of abdominal pain via modulation of visceral pain perception [28]. Due to its Generally Regarded As Safe (GRAS) status and the ability to survive transit through the digestive tract, $L$. acidophilus has been considered an ideal vehicle for mucosal-targeted delivery of vaccines and biotherapeutics $[29,30]$. As with other probiotic microbes, research has revealed in vivo mechanisms involved in their survival and interaction with the host to promote biodelivery and fitness in the gut. The presence of intact glycogen metabolic gene clusters in L. acidophilus NCFM and certain Lactobacillus species typically associated with natural or mammalian host environments led to our speculation that glycogen metabolism potentially contributes to the survival and probiotic functionalities of lactobacilli in the gastrointestinal (GI) tract.

This review highlights our recent findings on the genetics and physiology of glycogen metabolism in L. acidophilus [23], including factors (e. g. type and availability of carbon source, growth phase) which affect gene expression and glycogen biosynthesis, and the influence of glycogen metabolism on various probiotic-associated phenotypes of L. acidophilus. We also present our most current in vivo studies demonstrating that the capability of synthesizing intracellular glycogen contributes to the competitive retention of $L$. acidophilus in the mouse GI tract.

\section{Glycogen metabolic pathway genes among Lactobacillus}

The glycogen metabolic pathway in L. acidophilus is encoded by a $11.7-\mathrm{kb}$ chromosomal region consisting of $g \operatorname{lgBCDAP-amy-pgm}$ genes (LBA0680 to LBA0687) (Figure 2A) [23]. All seven genes are co-transcribed as a polycistronic mRNA and the gene cluster designated as the $g l g$ operon. To date, among the genome sequences of Lactobacillus species available in the NCBI genome database [http://www.ncbi.nlm.nih.gov/genome/ browse/], only $\sim 30 \%$ of the species possess complete glycogen metabolic gene sets. The $g l g$ operons identified in these Lactobacillus species have a conserved chromosomal mosaic arrangement of the glg genes, with the
$\operatorname{glgBCDAP}$ genes representing the core genes in these species (Figure 2B). In terms of sequence homology, the glycogen pathway enzymes of L. acidophilus exhibited 41 to $90 \%$ identity to the corresponding proteins among the other Lactobacillus species, with closest orthologs found in Lactobacillus amylovorus, Lactobacillus helveticus R0052, Lactobacillus delbrueckii subsp. bulgaricus PB2003/044-T3-4, L. delbrueckii subsp. lactis CRL581, Lactobacillus kefiranofaciens and Lactobacillus equicursoris. Phylogenetic analysis of the conserved glycogen synthase, GlgA, showed a phylogenetic relationship with resemblance to that of the $16 \mathrm{~S}$ rRNA gene sequences of Lactobacillus species [23]. This implied that the presence of this operon among specific lactobacilli was not due to recent horizontal gene acquisition.

The glg-encoding Lactobacillus species predominantly originate from mammalian hosts or natural environments, such as Lactobacillus salivarius, Lactobacillus ruminus, Lactobacillus plantarum, Lactobacillus rhamnosus, Lactobacillus gastricus, Lactobacillus mucosae and Lactobacillus murinus. Despite the general absence or degeneration of the gene cluster among the sequenced strains of L. helveticus and L. delbrueckii, both of which are commonly associated with domesticated dairy environments, the non-dairy origin strains L. helveticus R0052, a commercial probiotic strain isolated from sweet acidophilus milk [32], along with a human vaginal isolate of $L$. delbrueckii subsp. bulgaricus (PB2003/044-T3-4) and a cheese isolate of L. delbrueckii subsp. lactis (CRL581) appeared to harbor complete sets of glycogen metabolic pathway genes. This observation reflected a niche-specific function of glycogen metabolism among lactobacilli. The conservation of the genomic region flanking the glg operons in L. helveticus R0052 and $L$. acidophilus demonstrated that this operon was lost from the other sequenced strains of L. helveticus. In the case of L. delbrueckii subsp. bulgaricus, small remnants of the glg operon were found among the majority of the sequenced strains at the genomic region corresponding to that of the 


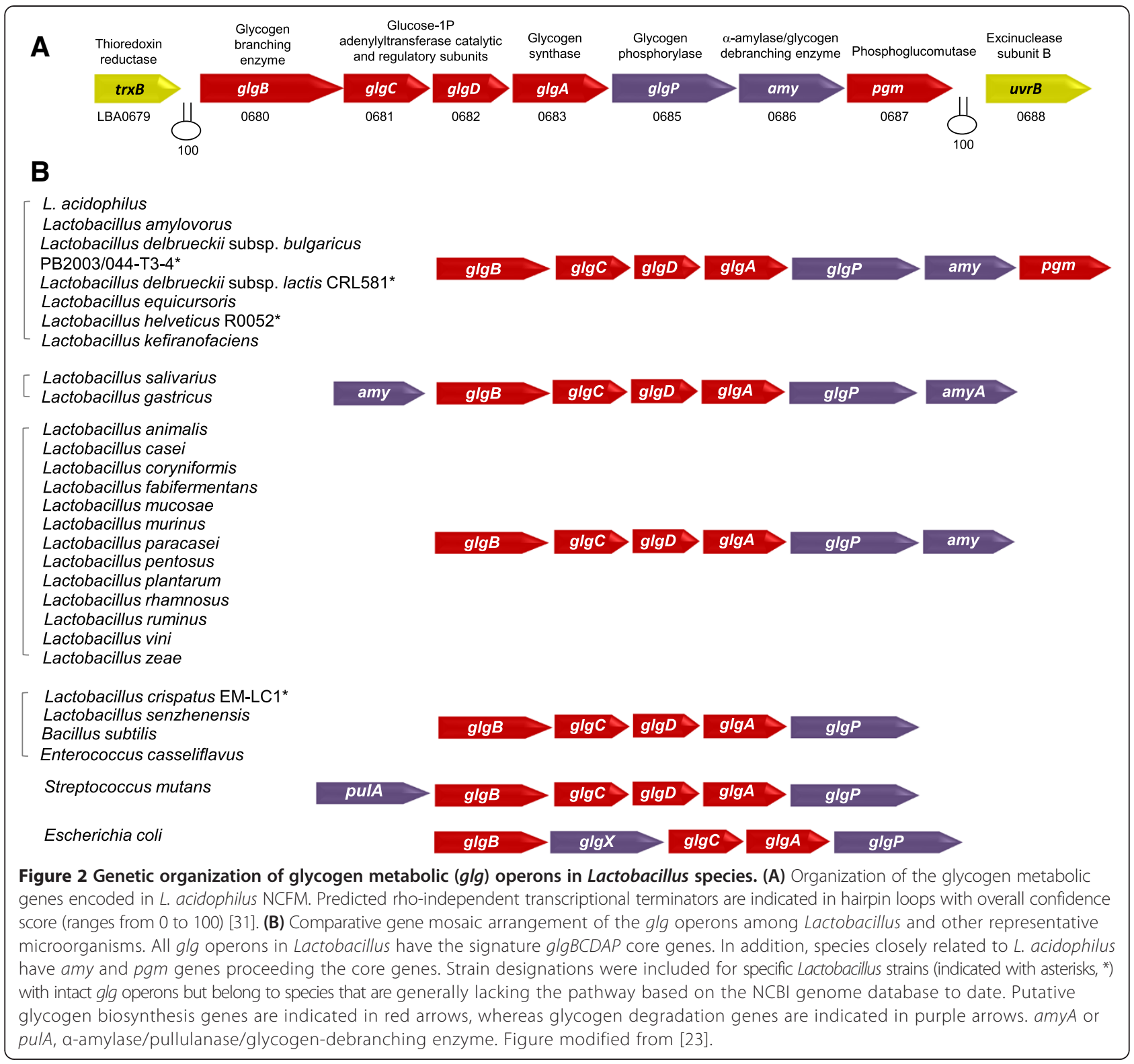

glg locus in strain PB2003/044-T3-4. This suggested that only the latter strain preserved an intact $g l g$ operon as part of a niche-specific gene repertoire equipped for its adaptation to the human vaginal environment.

It is noteworthy that, most Bifidobacterium species known as dominant GI commensals and probiotic microorganisms also possess all the essential enzymes for glycogen biosynthesis and catabolism [2]. The glycogen pathway genes in Bifidobacterium species, like in C. glutamicum [16], another species of Actinobacteria, are not organized as a single operon but instead are dispersed within the genomes [23]. Meanwhile, no glycogen metabolic genes were found in several species that are closely related to L. acidophilus and are commonly associated with mammalian host niches, notably Lactobacillus gasseri, Lactobacillus johnsonii and the majority of the Lactobacillus crispatus strains. Nonetheless, the presence of intact glycogen metabolism gene sets in L. acidophilus and other glg-encoding Lactobacillus species, in specific host-associated strains of $L$. helveticus and $L$. delbrueckii subsp. bulgaricus, as well as its prevalence in bifidobacteria, indicates a functional and ecological advantage conferred by their ability to synthesize glycogen.

\section{Glycogen metabolism in L. acidophilus is dependent on carbon source and growth phase}

The differential expression of the $g l g$ operon and glycogen accumulation profiles under various carbohydrate conditions demonstrated that glycogen biosynthesis in L. acidophilus is highly dependent on the type of sugar substrates present 
[23]. From a previous microarray-based global transcriptomic study of carbohydrate utilization by L. acidophilus, expression of the $g l g$ operon was upregulated when raffinose or trehalose was provided as a sole carbon source compared to other sugars; whereas glucose appeared to repress the glg genes [33]. Subsequently, real time-quantitative PCR (RT-qPCR) experiments and quantitative glycogen assays showed that raffinose was among the sugar substrates examined that induced the highest level of the $g l g$ operon expression and intracellular glycogen accumulation, followed by the disaccharides trehalose and lactose [23]. Both glg expression and glycogen biosynthesis were repressed by glucose. This is consistent with the identification of a catabolite response element (cre) upstream of the $g l g$ operon which is further evidence that glycogen metabolism is subject to catabolite regulation [33]. On the contrary, in microorganisms such as C. glutamicum, S. mutans and Salmonella enteritidis, glycogen accumulation and gene expression were induced in the presence of glucose $[16,18,19]$. From the perspective of niche adaptation in the small intestinal environment, we theorized that the presence of glucose may signal for nutrient abundance and ideal conditions for population expansion, leading to the prioritization of carbon flow towards glycolysis and other biosynthetic pathways [23]. When glucose is deprived, cells may respond by de-repressing the catabolic machinery for complex carbohydrates along with activating the glycogen metabolic pathway. Under these conditions, glycogen metabolism may generate energy storage reserves and serve as a carbon capacitor in order to regulate downstream metabolic flux, presumably in an energy conservation state to prolong survival.

Temporal glycogen accumulation profiling revealed significant differences during growth on raffinose or trehalose (Figure 3). On both sugars, the highest intracellular glycogen content was observed during early log phase, indicating that $L$. acidophilus was actively synthesizing and accumulating glycogen during the early growth phase. Previous observations in C. glutamicum also showed that glycogen accumulated rapidly at early log phase when growing on various sugar substrates [16]. While a dramatic decrease in glycogen level occurred in cells grown on trehalose following early log phase, the raffinose-grown cells maintained at about $50 \%$ of their initial glycogen level as the cells entered stationary phase, and remarkably, continued to retain a stable level throughout late stationary phase (Figure 3). Since the glycogen synthesis and degradation genes are co-transcribed, it is likely that the observed glycogen levels were the result of post-transcriptional regulation. It was previously proposed that the balance of parallel synthesis and degradation pathways serves to maintain the glycogen structures and function as a carbon capacitor for sensitive regulation of downstream carbon and energy fluxes $[6,11,12,34]$. It is plausible that $L$. acidophilus may maintain a higher level of intracellular glycogen as well as $g l g$ expression to enhance sustainability when growing on a more complex carbon source, a scenario most likely encountered in the gut environment. This presents a novel strategy for utilizing raffinose and potentially other prebiotic oligosaccharides to induce the cells into accumulating and maintaining a stable glycogen pool in order to enhance the viability and residence time of L. acidophilus in the GI tract. Meanwhile, it is interesting to note that the transcription of the $g l g$ operon was elevated in cells cultured in semi-defined medium without a carbon source [23] as well as during late stationary phase (Figure $3 \mathrm{~B}$ ). We speculated this might be partly due to the de-repression of the $g l g$ operon when fermentable carbon sources are depleted. It is also possible that the $g l g$ operon was induced during transient starvation conditions, presumably for the catabolism of remaining glycogen storage, or as part of a stress response regulon.

\section{Functional characterization of the $g / g$ operon}

An in-frame deletion of the $\operatorname{glg} A$ or $g \lg B$ gene resulted in a glycogen-deficient phenotype in both mutants of L. acidophilus (Figure 4). Hence, the inactivation of $\operatorname{glg} A$ or $\operatorname{glg} B$ alone abolished the ability of the mutants to synthesize glycogen, confirming that (a) the $g l g$ operon is functional in L. acidophilus; (b) $g \lg A$ and $g \lg B$ encodes for a glycogen synthase and a glycogen-branching enzyme, respectively, and (c) both functional $g \lg A$ and $g \lg B$ are required for the formation of intracellular glycogen. Interestingly, both $\triangle g l g A$ and $\triangle g l g B$ mutants, but not the $\triangle g l g P$ or $\triangle a m y$ mutant, exhibited less robust growth on raffinose compared to the parent strain [23]. These results suggest an interconnection between glycogen synthesis and raffinose metabolism. The growth defect on raffinose might be due to the major influx of glucose-1-phosphate intermediates (resulting from raffinose catabolism) into the glycogen synthesis pathway, leading to the accumulation of ADP-glucose and linear $\alpha$-glucan chains in the $\triangle g l g A$ and $\triangle g l g B$ mutants, respectively. The inability of the mutants to convert these intermediates into glycogen macromolecules may consequently affect carbon down flow and compromise growth.

Inactivation of the $\operatorname{glg} B$ or $g \lg P$ gene resulted in slightly slower growth and colony morphotype variation in MRS medium. Both mutants also showed increased sensitivity to bile and simulated small intestinal juice. The bile sensitivity phenotype might be attributed by an overall growth defect which led to decreased efficiency of the bile efflux systems [36], and possibly the functionality of other resistance mechanisms that protect the cells from digestive enzymes (i.e., amylase, protease, lipase) present in the simulated small intestinal juice. Given that the 


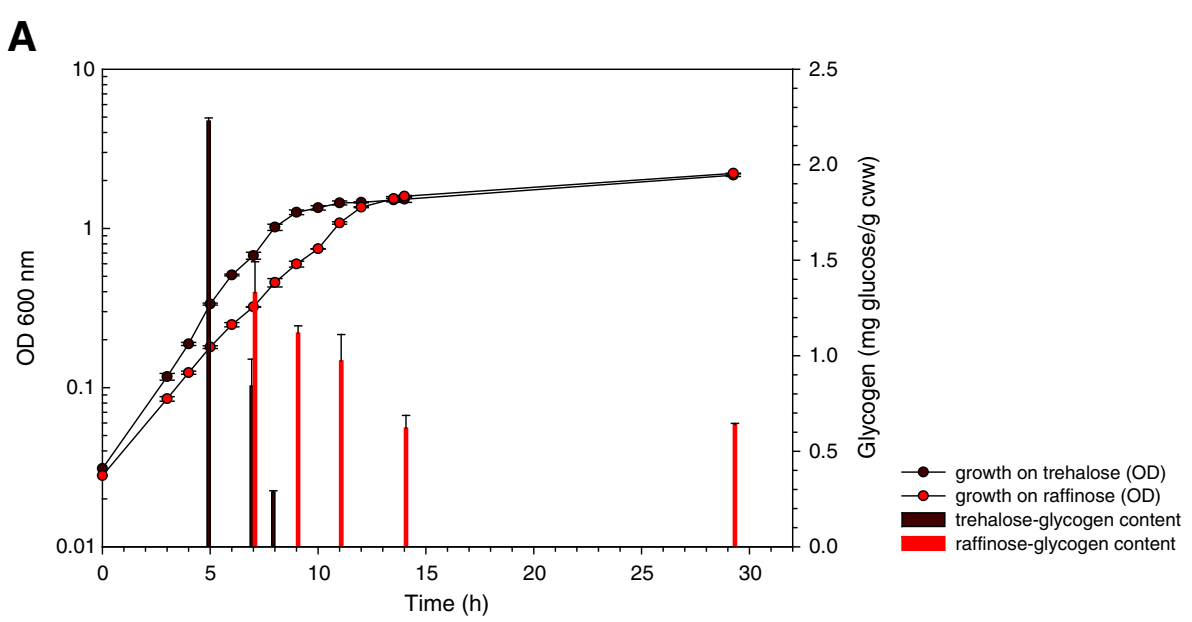

B

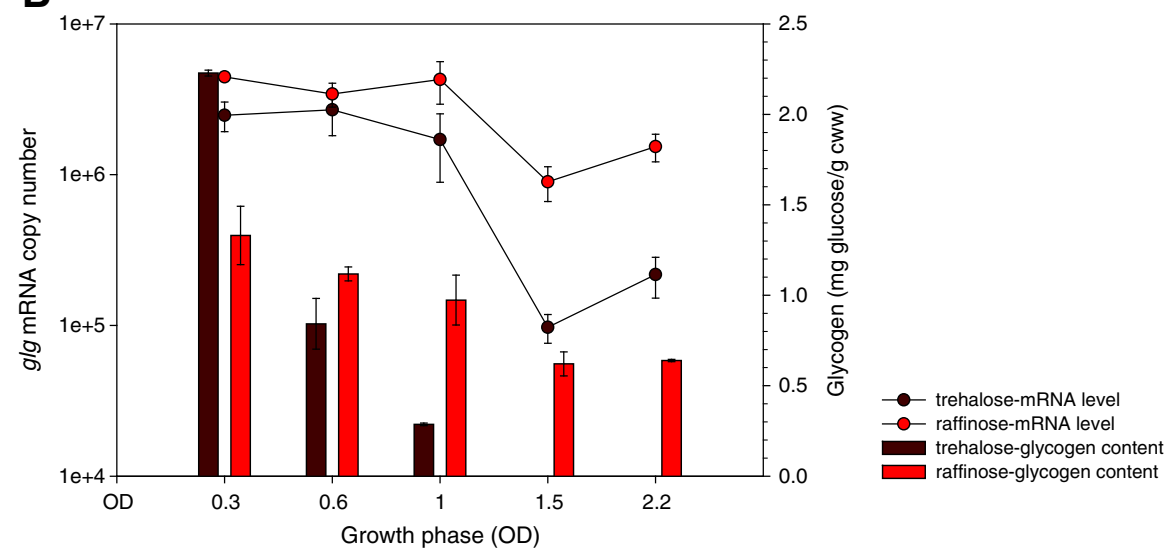

Figure 3 Intracellular glycogen levels and $g l g$ expression during growth on raffinose versus trehalose. (A) Growth and glycogen accumulation profiles of $L$. acidophilus in semi-defined medium (SDM) [35] containing $2 \%$ of raffinose or trehalose as the sole carbon source. The glycogen levels at various growth phases (indicated by $\mathrm{OD}_{600}$ ) from $(\mathbf{A})$ were compiled and plotted against the transcript levels of the glg operon in (B). The data represent the mean \pm standard deviation for two independent biological replicates. Growth phases are represented by $\mathrm{OD}_{600}: 0.3$, early log; 0.6 , mid-log; 1.0 , early stationary; 1.5 , stationary, and 2.2 , late stationary. Intracellular glycogen content was quantified by hexokinase/glucose-6-phosphate dehydrogenase-based glucose assay and expressed as mg of glucose (released from glycogen by amyloglucosidase) per $\mathrm{g}$ of cell wet weight (mg glucose/g cww). Figure adapted from [23].

same phenotype was not observed in the other glycogendeficient mutant, $\triangle g l g A$, we speculate that in the $\triangle g l g B$ mutant, accumulation of the $\alpha$-glucan polymers that were unable to form glycogen structures resulted in various physiological perturbations that affected its growth and survival in the small intestinal environment. In M. tuberculosis, inactivation of $\mathrm{GlgB}$ was lethal which might be due to the intracellular accumulation of poorly soluble linear $\alpha$-glucan polymers [14]. With respect to the $\Delta g l g P$ mutation, a similar growth defect and morphological alteration was also observed in $M$. smegmatis carrying a mutation in the glycogen-degrading enzyme gene, $g l g E$ [12]. Based on the authors' proposed model of glycogen metabolism as a carbon capacitor for glycolysis, the absence of GlgE disrupts glycogen recycling, resulting in glucose molecules sequestered in glycogen, thereby compromising downstream metabolic pathways. Hence, the growth and bile sensitivity phenotypes of the L. acidophilus $\Delta g l g P$ mutant emphasize the physiological importance of coordinated glycogen synthesis and degradation as well as the ability of the cells to retrieve a carbon source from glycogen storage during normal growth as well as stress conditions.

In vivo competitive and displacement colonization studies supporting the role of glycogen biosynthesis in gut retention

In enteric bacteria such as $E$. coli, glycogen storage plays a critical role in the colonization of the mouse GI tract as evidenced by the significant colonization defect of mutants that are unable to synthesize or degrade internal glycogen [15]. In order to establish whether the ability to synthesize intracellular glycogen contributes to the retention of $L$. acidophilus in the GI environment, 


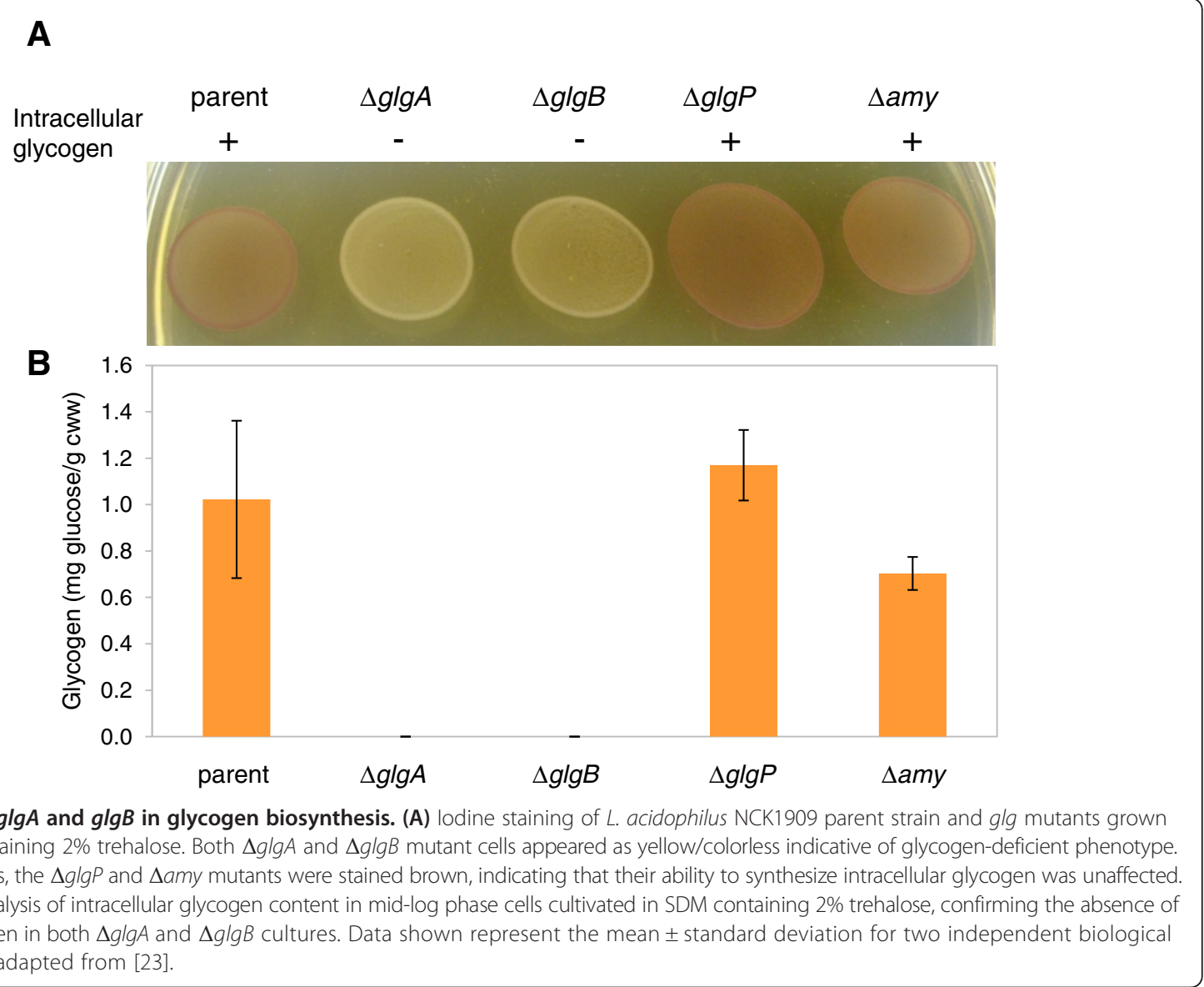

we conducted two in vivo studies using a $129 \mathrm{~S} 6 / \mathrm{SvEv}$ germ-free mouse model. The first experiment involved a competitive colonization strategy whereby the germ-free mice were co-colonized with both the $\triangle g l g A$ glycogendeficient mutant and the parent strain in equal ratios. The GI retention of both strains was monitored by plating fecal samples during a period of 4 weeks (Figure 5). In the follow-up experiment, germ-free mice were first mono-colonized with the $\triangle g l g A$ mutant, followed by subsequent introduction of the parent strain into the same mice to determine whether the ability to generate glycogen reserves would enable the parent population to overturn the dynamic of the established mutant population in the gut.

Prior to the mouse experiment, in order to enable differential enumeration and comparison of the bacterial populations representing the parent (NCK1909) [37] and the $\triangle g l g A$ (NCK2180) [23] strains in the fecal samples, the parent and mutant strains were marked with spontaneous resistance to different antibiotics. Both antibiotics, rifampicin (Rif) and streptomycin (Str), were selected for use due to their low minimal inhibitory concentrations [38] and relatively low spontaneous resistance frequency exhibited by $L$. acidophilus. Both antibiotic resistance phenotypes do not provide cross-protection against each other. One Rif-resistant $\left(\mathrm{Rif}^{\mathrm{R}}\right)$ parent and one Str-resistant $\left(\mathrm{Str}^{\mathrm{R}}\right) \Delta g \lg A$ mutant colonies were isolated from exposure to the respective antibiotics and designated as NCK2300 (parent) and NCK2301 ( $\triangle g l g A$ mutant), respectively (Figure 5A and Additional file 1). Both antibiotic-resistant derivatives exhibited undistinguishable growth phenotypes compared to their native counterparts, and the antibiotic resistance phenotypes were stable throughout 30 consecutive passages in the absence of antibiotics, confirming that the spontaneous antibiotic resistant phenotype was stable and did not negatively impact growth (Additional file 1). Since the spontaneous antibiotic resistance phenotype, once established, is inherent in the selected strains, this strategy eliminates the need of external antibiotic agents for maintenance of resistance phenotypes within the bacterial populations in an in vivo system. It also allows for the co-investigation of two or more bacterial strains competitively, in vivo.

\section{Competitive co-colonization of glycogen-deficient $\triangle g l g A$ mutant and parent strains}

On the initial day (Day 0) of the mouse experiment, both parent (NCK2300) and $\triangle g l g A$ mutant (NCK2301) derivatives 


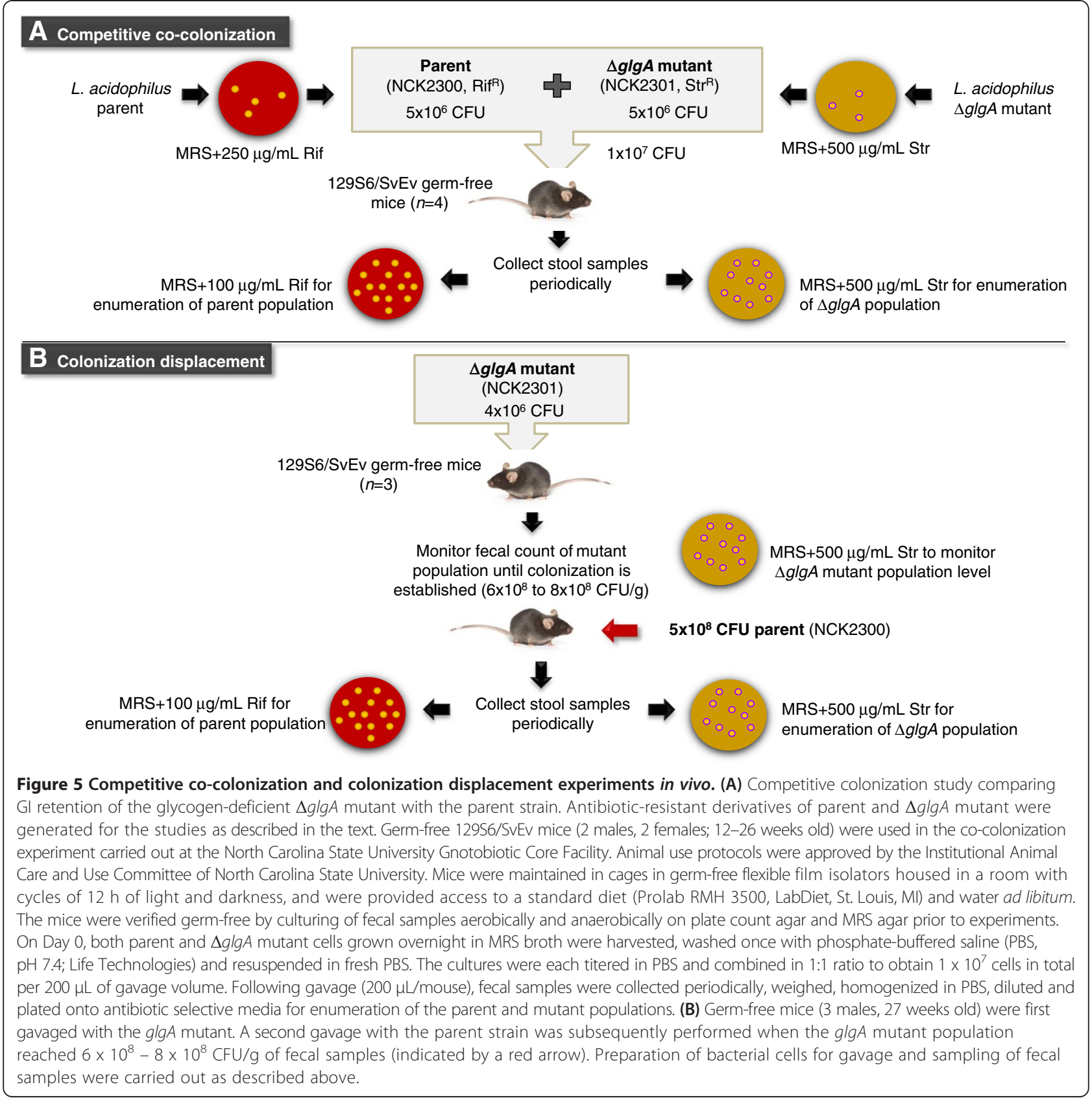

were combined in 1:1 ratio of cell concentrations and delivered to the germ-free mice $(n=4)$ by intragastric gavage $\left(1 \times 10^{7} \mathrm{CFU}\right.$ total in $\left.200 \mu \mathrm{l}\right)$ (Figure 5A). Following administration, fecal samples were collected periodically to differentially enumerate the parent and mutant populations on antibiotic selective media. The results in Figure 6A showed an initial population decline of the $\Delta g l g A$ mutant on Day 4 post-gavage. The population level of the mutant continued to decline and was maintained at a $2 \log$ reduction compared to the parent population. We suspect that the lack of a further decline of the mutant population was likely due to the coprophagic nature of rodents. This study demonstrated that the ability of L. acidophilus to synthesize intracellular glycogen storage provides a competitive advantage in the mouse GI tract.

\section{Colonization displacement of $\triangle g l g A$ mutant by the parent strain}

Germ-free mice $(n=3)$ were initially mono-colonized with the $\lg A$ mutant by intragastric gavage at a dose of ca. $4 \times$ $10^{6} \mathrm{CFU}$ (Figure 5B). In the absence of competition, the mutant was able to establish initial population levels in the gut (> $6 \times 10^{8} \mathrm{CFU} / \mathrm{g}$ of fecal samples; Figure $6 \mathrm{~B}$ ) that are at least 5 times higher than its population levels during 

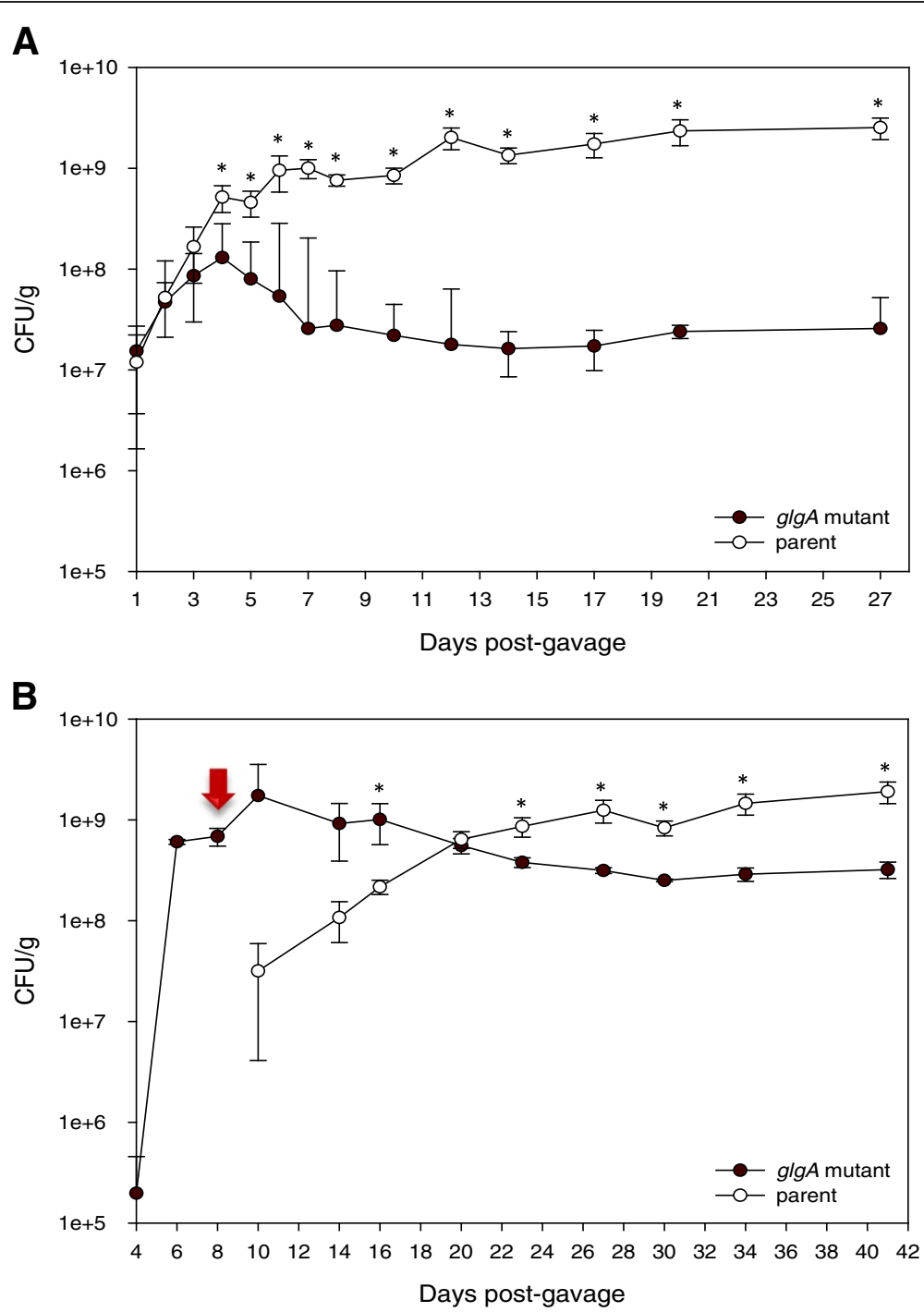

Figure 6 Co-colonization and colonization displacement studies demonstrating the role of glycogen biosynthesis on competitive gut retention. (A) Co-colonization of both the glycogen-deficient $\Delta g / g A$ mutant and the parent strain in germ-free 129S6/SvEv mice resulted in an overall 2 log reduction of the $\Delta g / g A$ mutant population. Data shown represent the median cell counts values and mean cell counts \pm standard deviation from all four mice. (B) Addition of the parent strain to gnotobiotic mice previously mono-colonized with the $\triangle g l g A$ mutant resulted in a population shift with gradual displacement of the mutant population by the parent. Red arrow indicates the timepoint at which the parent strain was administered in a single gavage dose of $5 \times 10^{8} \mathrm{CFU}$. Data shown represent the average cell counts values and mean cell counts \pm standard deviation from all three mice. An asterisk $\left.*^{*}\right)$ indicates a statistically significant difference between the mutant and parent populations $(p$-value $<0.05)$. Both in vivo studies established that a functional glycogen biosynthetic pathway contributes to the competitive advantage and retention of $L$. acidophilus in the $\mathrm{Gl}$ tract.

previous co-colonization studies (highest population count $\sim 1 \times 10^{8} \mathrm{CFU} / \mathrm{g}$; Figure 6A). The parent strain (ca. $5 \times 10^{8} \mathrm{CFU}$ ) was subsequently administered to the mice by gavage when the $\operatorname{glg} A$ mutant population reached the level of $6 \times 10^{8}-8 \times 10^{8} \mathrm{CFU} / \mathrm{g}$. The population dynamic of the mutant and parent were monitored as described previously by differential plating of fecal samples on antibiotic selective media. Our data showed that the introduction of the parent strain resulted in a population shift whereby the decline in the mutant abundance coincides with the continual increase of the parent population level (Figure 6B). The observed population displacement of the $\triangle g l g A$ mutant by the parent strain further substantiates the role of glycogen metabolism on the competitive fitness of $L$. acidophilus in the host environment. Moreover, the ability of the $\triangle g l g A$ mutant to establish initial colonization in a germ-free system provided evidence that the decline of the mutant 


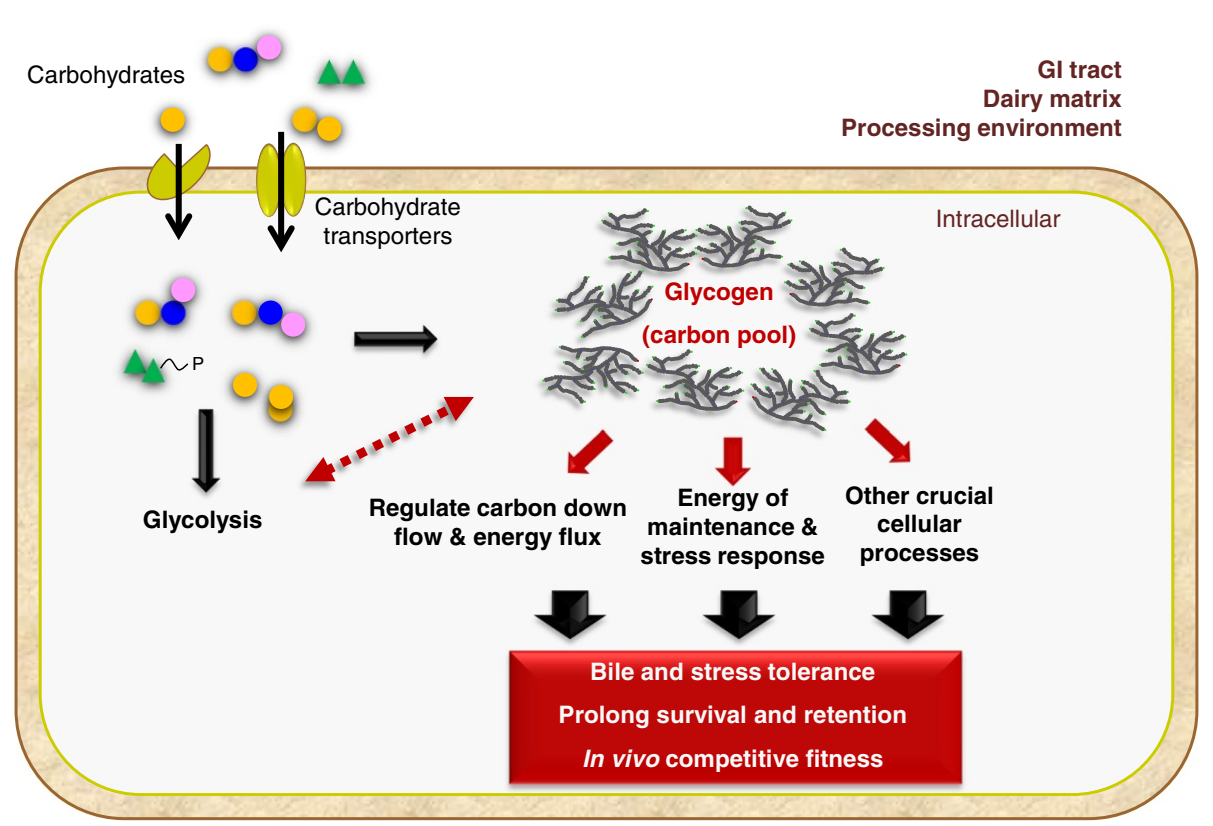

Figure 7 Proposed mechanisms and functions of glycogen metabolic pathway in L. acidophilus. Based on proposed central functions of glycogen metabolism in other microorganisms $[6,11,12]$ and findings from this study, carbohydrate substrates imported by the cells may be either metabolized through the glycolytic pathway, or shunted to glycogen biosynthetic pathway when a carbon source is not immediately required for glycolysis. The carbon pool may serve as an energy storage reserve and as a carbon capacitor that senses and modulates downstream carbon flow to maintain efficient carbon utilization and energetic homeostasis of the cells. The roles of glycogen metabolism in central carbon metabolism influence various physiological functions and consequently the retention and probiotic attributes of $L$. acidophilus. Our fundamental understanding of this pathway will inspire strategies to improve the stability and functionalities of probiotic and beneficial commensal microorganisms in the host.

population in the initial competitive colonization study was not due to colonization defect in vivo, but rather the lost of competitiveness against the parent population that possesses glycogen biosynthetic capability.

\section{Conclusions}

These studies have provided crucial insights into the broad biological functions and probiotic attributes in Lactobacillus that rely on functional glycogen biosynthetic and catabolic pathways. Genetic dissection of the pathway in $L$. acidophilus has thus far suggested that glycogen metabolism plays multifaceted roles including normal growth maintenance, the utilization of certain complex sugars (e.g. raffinose), bile tolerance and competitive fitness in the gut. Although the underlying mechanisms on how glycogen metabolism operates globally remains to be established, it is likely that the in vitro phenotypes translated to the observed competitiveness of L. acidophilus, in vivo. Koch [39] previously proposed that bacteria in nature generally exist between the states of feast or famine whereby nutrients are rarely in constant supply. This is supported by the fact that glycogen storage is ubiquitous among enteric bacteria, possibly due to the necessity to support rapid growth in the intestinal environment where there is intense competition for nutrients [15]. In view of this, the ability of L. acidophilus to synthesize and store energy in the form of glycogen, either prior to (e.g. during commercial processing or product storage) or during its transit through the host, potentially confers advantages to its retention and probiotic activities in the GI tract. We further postulate that the concerted glycogen anabolism and catabolism in L. acidophilus serves to regulate central carbon flow and operate based on nutrient status to conserve energy and resources in the competitive intestinal environment (Figure 7). Overall, we foresee that this work will serve as a foundation to expand our understanding on the roles of glycogen metabolism in other probiotic and commensal species of Lactobacillus and Bifidobacterium, and to apply the knowledge for improvement of biodelivery and gut persistence of these microorganisms within the host.

\section{Additional file}

Additional file 1: Methods on generation of spontaneous antibiotic-resistant derivatives of $L$. acidopohilus parent and $\Delta g l g A$ glycogen-deficient mutant for in vivo studies, and Figure depicting 
growth and stability assessment of the antibiotic-resistant phenotypes of NCK2300 (parent) and NCK2301 ( $\Delta$ glgA mutant).

\section{Abbreviations}

Gl: Gastrointestinal; GlgA: Glycogen synthase; GlgB: Glycogen-branching enzyme; GlgCD: Glucose-1-phosphate adenylyltransferase; GlgP: Glycogen phosphorylase; GlgX: Glycogen-debranching enzyme; Pgm: Phosphoglucomutase; Amy: Putative a-amylase/debranching enzyme; Rif: Rifampicin; Str: Streptomycin; CFU: Colony forming unit.

\section{Competing interests}

The authors declare that they have no competing interests.

\section{Authors' contributions}

YJG conceived the study, designed and performed the in vivo murine experiments, and wrote the manuscript. TRK participated in the study design and coordination. Both authors read and approved the final manuscript.

\section{Acknowledgements}

This work was supported in part by the North Carolina Agricultural Foundation and Danisco/DuPont Nutrition \& Health USA, Inc. We express our gratitude to Dr. Sarah O'Flaherty, Dr. Sue Tonkonogy, Rosemary SanozkyDawes and Emma Call for technical advice and insightful discussion. Our appreciation also extends to Ashley Weaver from the Gnotobiotic Core at the College of Veterinary Medicine, North Carolina State University for assistance with experiments using germ-free and gnotobiotic mice. The Gnotobiotic Core is a facility of the Center for Gastrointestinal Biology and Disease, funded by NIH grant P30 DK034987. This paper was contributed to the supplement for the Proceedings of the 11th International Symposium on Lactic Acid Bacteria (http://www.microbialcellfactories.com/supplements/13/S1).

Received: 1 May 2014 Accepted: 20 June 2014

Published online: 20 November 2014

\section{References}

1. Preiss J: Glycogen biosynthesis. In Encyclopedia of microbiology (Schaechter Med. 3rd edition. Oxford: Elsevier; 2009:145-158.

2. Wang L, Wise MJ: Glycogen with short average chain length enhances bacterial durability. Naturwissenschaften 2011, 98:719-729.

3. Wilson WA, Roach PJ, Montero M, Baroja-Fernandez E, Munoz FJ, Eydallin G, Viale AM, Pozueta-Romero J: Regulation of glycogen metabolism in yeast and bacteria. FEMS Microbiol Rev 2010, 34:952-985.

4. Preiss J: Bacterial glycogen synthesis and its regulation. Annu Rev Microbiol 1984, 38:419-458.

5. Alonso-Casajus N, Dauvillee D, Viale AM, Munoz FJ, Baroja-Fernandez E, Moran-Zorzano MT, Eydallin G, Ball S, Pozueta-Romero J: Glycogen phosphorylase, the product of the glgP gene, catalyzes glycogen breakdown by removing glucose units from the nonreducing ends in Escherichia coli. J Bacteriol 2006, 188:5266-5272.

6. Dauvillee D, Kinderf IS, Li Z, Kosar-Hashemi B, Samuel MS, Rampling L, Ball S, Morell MK: Role of the Escherichia coli glgX gene in glycogen metabolism. J Bacteriol 2005, 187:1465-1473.

7. Eydallin G, Viale AM, Moran-Zorzano MT, Munoz FJ, Montero M, Baroja-Fernandez E, Pozueta-Romero J: Genome-wide screening of genes affecting glycogen metabolism in Escherichia coli K-12. FEBS Lett 2007, 581:2947-2953.

8. Eydallin G, Montero M, Almagro G, Sesma MT, Viale AM, Munoz FJ, Rahimpour M, Baroja-Fernandez E, Pozueta-Romero J: Genome-wide screening of genes whose enhanced expression affects glycogen accumulation in Escherichia coli. DNA Res 2010, 17:61-71.

9. Kiel JA, Boels JM, Beldman G, Venema G: Glycogen in Bacillus subtilis: molecular characterization of an operon encoding enzymes involved in glycogen biosynthesis and degradation. Mol Microbiol 1994, 11:203-218.

10. Tzvetkov M, Klopprogge C, Zelder O, Liebl W: Genetic dissection of trehalose biosynthesis in Corynebacterium glutamicum: inactivation of trehalose production leads to impaired growth and an altered cell wall lipid composition. Microbiology 2003, 149:1659-1673.

11. Seibold GM, Eikmanns BJ: The glgX gene product of Corynebacterium glutamicum is required for glycogen degradation and for fast adaptation to hyperosmotic stress. Microbiology 2007, 153:2212-2220.
12. Belanger AE, Hatfull GF: Exponential-phase glycogen recycling is essential for growth of Mycobacterium smegmatis. J Bacteriol 1999, 181:6670-6678.

13. Busuioc M, Mackiewicz K, Buttaro BA, Piggot PJ: Role of intracellular polysaccharide in persistence of Streptococcus mutans. J Bacteriol 2009, 191:7315-7322.

14. Sambou T, Dinadayala P, Stadthagen $G$, Barilone N, Bordat $Y$, Constant $P$, Levillain F, Neyrolles O, Gicquel B, Lemassu A, Daffe M, Jackson M: Capsular glucan and intracellular glycogen of Mycobacterium tuberculosis: biosynthesis and impact on the persistence in mice. Mol Microbiol 2008, 70:762-774.

15. Jones SA, Jorgensen M, Chowdhury FZ, Rodgers R, Hartline J, Leatham MP Struve C, Krogfelt KA, Cohen PS, Conway T: Glycogen and maltose utilization by Escherichia coli 0157: $\mathrm{H7}$ in the mouse intestine. Infect Immun 2008, 76:2531-2540.

16. Seibold G, Dempf S, Schreiner J, Eikmanns BJ: Glycogen formation in Corynebacterium glutamicum and role of ADP-glucose pyrophosphorylase. Microbiology 2007, 153:1275-1285.

17. Dalmasso M, Aubert J, Even S, Falentin H, Maillard MB, Parayre S, Loux V, Tanskanen J, Thierry A: Accumulation of intracellular glycogen and trehalose by Propionibacterium freudenreichii under conditions mimicking cheese ripening in the cold. Appl Environ Microbiol 2012, 78:6357-6364.

18. Bonafonte MA, Solano C, Sesma B, Alvarez M, Montuenga L, Garcia-Ros D, Gamazo C: The relationship between glycogen synthesis, biofilm formation and virulence in Salmonella enteritidis. FEMS Microbiol Lett 2000, 191:31-36.

19. Spatafora G, Rohrer K, Barnard D, Michalek S: A Streptococcus mutans mutant that synthesizes elevated levels of intracellular polysaccharide is hypercariogenic in vivo. Infect Immun 1995, 63:2556-2563.

20. Harris GS, Michalek SM, Curtiss R 3rd: Cloning of a locus involved in Streptococcus mutans intracellular polysaccharide accumulation and virulence testing of an intracellular polysaccharide-deficient mutant. Infect Immun 1992, 60:3175-3185.

21. Suzuki E, Ohkawa H, Moriya K, Matsubara T, Nagaike Y, Iwasaki I, Fujiwara S, Tsuzuki M, Nakamura Y: Carbohydrate metabolism in mutants of the cyanobacterium Synechococcus elongatus PCC 7942 defective in glycogen synthesis. Appl Environ Microbiol 2010, 76:3153-3159.

22. Bourassa $L$, Camilli A: Glycogen contributes to the environmental persistence and transmission of Vibrio cholerae. Mol Microbiol 2009, 72:124-138.

23. Goh YJ, Klaenhammer TR: A functional glycogen biosynthesis pathway in Lactobacillus acidophilus: expression and analysis of the glg operon. Mol Microbiol 2013, 89:1187-1200.

24. Sanders ME, Klaenhammer TR: Invited review: the scientific basis of Lactobacillus acidophilus NCFM functionality as a probiotic. J Dairy Sci 2001, 84:319-331.

25. Kim HS, Gilliland SE: Lactobacillus acidophilus as a dietary adjunct for milk to aid lactose digestion in humans. J Dairy Sci 1983, 66:959-966.

26. Leyer GJ, Li S, Mubasher ME, Reifer C, Ouwehand AC: Probiotic effects on cold and influenza-like symptom incidence and duration in children. Pediatrics 2009, 124:e172-179.

27. Konstantinov SR, Smidt H, de Vos WM, Bruijns SC, Singh SK, Valence F, Molle D, Lortal S, Altermann E, Klaenhammer TR, van Kooyk Y: S layer protein A of Lactobacillus acidophilus NCFM regulates immature dendritic cell and T cell functions. Proc Natl Acad Sci U S A 2008, 105:19474-19479.

28. Rousseaux C, Thuru X, Gelot A, Barnich N, Neut C, Dubuquoy L, Dubuquoy C, Merour E, Geboes K, Chamaillard M, Ouwehand A, Leyer G, Carcano D, Colombel JF, Ardid D, Desreumaux P: Lactobacillus acidophilus modulates intestinal pain and induces opioid and cannabinoid receptors. Nat Med 2007, 13:35-37.

29. Mohamadzadeh M, Duong T, Sandwick SJ, Hoover T, Klaenhammer TR: Dendritic cell targeting of Bacillus anthracis protective antigen expressed by Lactobacillus acidophilus protects mice from lethal challenge. Proc Natl Acad Sci U S A 2009, 106:4331-4336.

30. Kajikawa A, Zhang L, Long J, Nordone S, Stoeker L, LaVoy A, Bumgardner S, Klaenhammer T, Dean G: Construction and immunological evaluation of dual cell surface display of HIV-1 Gag and Salmonella enterica serovar Typhimurium FliC in Lactobacillus acidophilus for vaccine delivery. Clin Vaccine Immunol 2012, 19:1374-1381.

31. Kingsford CL, Ayanbule K, Salzberg SL: Rapid, accurate, computational discovery of Rho-independent transcription terminators illuminates their relationship to DNA uptake. Genome Biol 2007, 8:R22. 
32. Tompkins TA, Barreau G, Broadbent JR: Complete genome sequence of Lactobacillus helveticus R0052, a commercial probiotic strain. J Bacteriol 2012, 194:6349.

33. Barrangou R, Azcarate-Peril MA, Duong T, Conners SB, Kelly RM, Klaenhammer TR: Global analysis of carbohydrate utilization by Lactobacillus acidophilus using cDNA microarrays. Proc Natl Acad Sci U S A 2006, 103:3816-3821.

34. Shim JH, Park JT, Hong JS, Kim KW, Kim MJ, Auh JH, Kim YW, Park CS, Boos W, Kim JW, Park KH: Role of maltogenic amylase and pullulanase in maltodextrin and glycogen metabolism of Bacillus subtilis 168. J Bacteriol 2009, 191:4835-4844.

35. Kimmel SA, Roberts RF: Development of a growth medium suitable for exopolysaccharide production by Lactobacillus delbrueckii ssp. bulgaricus RR. Int J Food Microbiol 1998, 40:87-92.

36. Pfeiler EA, Klaenhammer TR: Role of transporter proteins in bile tolerance of Lactobacillus acidophilus. Appl Environ Microbiol 2009, 75:6013-6016.

37. Goh YJ, Azcarate-Peril MA, O'Flaherty S, Durmaz E, Valence F, Jardin J, Lortal S, Klaenhammer TR: Development and application of a upp-based counterselective gene replacement system for the study of the S-layer protein SlpX of Lactobacillus acidophilus NCFM. Appl Environ Microbiol 2009, 75:3093-3105.

38. D'Aimmo MR, Modesto M, Biavati B: Antibiotic resistance of lactic acid bacteria and Bifidobacterium spp. isolated from dairy and pharmaceutical products. Int J Food Microbiol 2007, 115:35-42.

39. Koch AL: The adaptive responses of Escherichia coli to a feast and famine existence. Adv Microb Physiol 1971, 6:147-217.

doi:10.1186/s12934-014-0094-3

Cite this article as: Goh and Klaenhammer: Insights into glycogen metabolism in Lactobacillus acidophilus: impact on carbohydrate metabolism, stress tolerance and gut retention. Microbial Cell Factories 2014 13:94.

\section{Submit your next manuscript to BioMed Central and take full advantage of:}

- Convenient online submission

- Thorough peer review

- No space constraints or color figure charges

- Immediate publication on acceptance

- Inclusion in PubMed, CAS, Scopus and Google Scholar

- Research which is freely available for redistribution 